\title{
REMARKS ON DEEP INELASTIC SCATTERING
}

\author{
M. KLEIN \\ Deutsches Elektronensynchrotron \\ Platanenallee 6, \\ 15738 Zeuthen, Germany \\ E-mail:klein@ifh.de
}

These remarks are devoted to the history of the physics of deep inelastic lepton nucleon scattering, to selected highlights at the DIS06 workshop and to developments towards a future of the field and its relations to the LHC.

\section{Preface}

Deep inelastic lepton-nucleon scattering (DIS) investigates the electromagnetic, weak and strong interactions, the dynamics of partons in the nucleon and it searches for new physics in the lepton-quark sector. As a particularly clean process which involves the leptonic probe and a hadron in the initial state, DIS provides unique results, in particular on the strong interaction dynamics, and thus it is an inherent part of high energy physics. The current frontier of DIS is at HERA, which resolves proton's structure down to $10^{-18} \mathrm{~m}$. Despite its most impressive results, which have been obtained until today in experimental and theoretical investigations, DIS tends to be forgotten when planning for a future of particle physics, and HERA will be shut down years too early. The recent generation of colliders at the Fermi energy scale, in $e^{+} e^{-}$(LEP), $p \bar{p}$ (TeVatron) and ep (HERA), all failed to find departures from the standard model, in terms of new particles or interactions. Hopes for future discoveries are mostly directed to $p p$ (LHC) and $e^{+} e^{-}$(ILC) interactions at the TeV scale, with ep sometimes ignored. While this phenomenon is not new ${ }^{a}$ it still is remarkable and has led me to make a small detour into the past when given the task of summarising the workshop on DIS at Japan in April 2006. Usually one would attempt to pre-

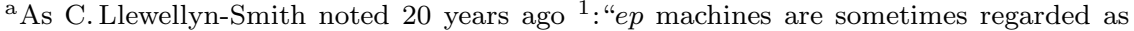
poor sisters of $e^{+} e^{-}$and $p p$ machines, but we should remember the story of Cinderella: poor sisters may strike rich.." 
dict what may be highlights at the following big summer conference. Before attempting this for ICHEP06, in Section 2, I revisited previous Rochester Conferences in order to perhaps learn lessons about the role of DIS and the way the international community has been confronted with its results and plans. Thus this summary begins, Section 1, with observations documented in the Proceedings, not referenced here for space reasons, of the ICHEP conferences in 1956, 66, 76, 86 and 96. Finally, in Section 3, a few remarks are made on theoretical questions. In many respects this summary is complementary to the ones of previous DIS Workshops by Alan Martin (2004) and Alan Caldwell (2005).

\section{Past}

The 1956 Rochester Conference on "High Energy Nuclear Physics" had a theoretical session with F. Dyson presiding in which, among others, talks were given by Gell-Mann on "Dispersion relations in $\pi N$ and $\gamma N$ scattering" and by V. Weisskopf on "A Chew-Low treatment of S-wave meson-nucleon scattering". Modern quantum field theory and the standard model (SM) seemed far away. In session IX on "Mesonic Atoms, $e N$ and $\gamma N$ Scattering and Miscellaneous Topics" a talk was given by R. Hofstadter on "Scattering of high energy electrons from hydrogen, deuterium and helium". The talk presented an update of results which were presented a year before, at the Vth Annual Rochester Conference at which Hofstadter was scheduled to talk in session VII on "Accelerator Physics". The summary notes a "presentation of some of the elegant electron-scattering work done by a large group" of seven physicists. The scattered electron angular distribution analysis suggested a finite proton radius of $(7.0 \pm 2.4) \cdot 10^{-14} \mathrm{~cm}$ assuming the "charge and the moment are diffused over the same volume", noting also that the separation of the two finite-size effects was possible from measurements at a variety of energies. It thus seems that our field was born 50 years ago. Interestingly, one of the earliest ideas was to disentangle the two formfactors $\left(G_{E}, G_{M}\right.$ or $\nu W_{2}, W_{1}$ or $\left.F_{2}, F_{L}\right)$ by changing the beam energies. It certainly was one of the highlights of this years DIS workshop when the two HERA collider experiments, H1 and ZEUS, expressed a firm interest in measuring $F_{L}$ with a low energy run at HERA.

Ten years later, at the 1966 "International Conference on High-Energy Physics" S. Drell reported on "Electrodynamic Interactions" presenting form factor measurements from DESY, data on $e p \rightarrow e \pi n$, on multi-electron production or charge asymmetry measurements at $Q^{2} \simeq 0.5 \mathrm{GeV}^{2}$ from 
Cornell and elsewhere. In that year the 2mile SLAC Linac started to operate. "I'd very much like to see", Drell added to his own talk, "inelastic electron or muon cross sections measured; they provide the inelastic form factors that are of great interest in their own right", mentioning $n-p$ mass difference calculations, spin and the hyperfine-structure and Bjorken's sum rules and asymptotic predictions. Soon afterwards the $Q^{2}$ independence of $\nu W_{2}$ at $\omega=1 / x \simeq 5$ observed at SLAC provided evidence for a partonic structure of the proton while subsequent data on $R=\sigma_{L} / \sigma_{T}$ determined the quark spin to be $1 / 2$. Gell-Mann in his opening talk at the Conference argued for focussing efforts on a next "higher-energy accelerator so that we can do more experiments over the next generation and really learn more about the basic structure of matter" while M. Froissart presented a "Logical map of 'Fundamental' concepts" which included many more than seem to have survived today. Weak interactions had come forward and particle physics was approaching the birth of the SM, to which DIS had contributed one of the most important ingredients with the discovery of quarks.

The XVIII ICHEP Conference at Tbilissi in 1976 celebrated the discovery of open charm as its highlight. DIS had made major progress with a new generation of higher energy fixed target neutrino and muon scattering experiments. Charged and neutral current data were presented by B. Barish et al. and the experiment of $\mathrm{H}$. Anderson showed a measurement of $F_{2}$ at $Q^{2} \simeq 6 \mathrm{GeV}^{2}$ which, on a linear $x$ scale extending to about 0.01 , showed no tendency for $F_{2}\left(x, Q^{2}\right)$ to not rise towards low $x$. V. Zakharov concluded that the parton model was working "not bad indeed", asymptotic freedom "may show up" and "the overall picture has not yet settled but may be simple". A. Salam argued that leptons may "share with hadrons the strong force" as the electromagnetic, weak and gravitational force. "Dogmas", he remarked, "are absolutely essential for the progress of science but they become tragic if they succeed in stopping experimentation designed to prove them wrong". The hope to find resonant electron-quark states hasn't been fulfilled so far but new attempts to reach higher energy, as the LHeC ${ }^{b}$ presented to this workshop are worthy of careful evaluation.

In 1986 DIS contributions were made to ICHEP XXIII, with high statistics data on $F_{2}$ measured by BCDMS in $\mu C$ scattering and on the nuclear dependence of $R$. As cited above quite some attention was given to the

\footnotetext{
${ }^{\mathrm{b}}$ It has been proposed to add a new technology electron ring to the LHC and build a Large Electron Hadron Collider (LHeC) ${ }^{3}$ as an $e p$ machine of energy of about $E_{e}=70 \mathrm{GeV}$ and $E_{p}=7000 \mathrm{GeV}$ at an anticipated high luminosity of $10^{33} \mathrm{~cm}^{-2} \mathrm{~s}^{-1}$.
} 
future of HEP. Reading Ref. ${ }^{1}$ one notes how high the hopes were to find a light Higgs and a supersymmetric particle spectrum at the forthcoming colliders at the Fermi scale. Similarly remarkable is the experimental progress which was made in the 20 years since ${ }^{1}$ on the construction and design of high luminosity machines and in the ability to operate tracking detectors near the beam line. Reminiscent to today's discussion about the importance of having 1 or $3 \mathrm{TeV}$ of energy in the ILC or CLIC, in 1986 one discussed the LHC vs. the SSC. It is curious to note that the SSC went ahead but was stopped while the lower energy $p p$ collider LHC now approaches completion. The linear collider future depends on the LHC results, so does a new $e p$ collider, mutatis mutandis.

A further ten years later, at the Rochester Conference at Warsaw, 1996, DIS attracted large attention because the first accurate measurements from HERA appeared, such as a $5 \%$ measurement of the rise of $F_{2}$ towards low $x$. In view of what later was called "HERA III" ${ }^{4}, \mathrm{~B}$. Wiik noted in the 1996 HERA Workshop proceedings that "we have just scratched the surface of HERA physics". The SMC Collaboration presented the $x$ distribution of spin for different quark species which showed the up-valence spin to be aligned with the proton spin, $x \Delta u_{v}>0$, the down-valence spin to be antiparallel, $x \Delta d_{v}<0$, yet compatible with zero, and the antiquarks likely carrying no spin, results which have been confirmed with high accuracy and including the strange quark by the HERMES Collaboration recently. At Warsaw COMPASS was reported to be recommended to CERN to study the gluon spin contribution, $\Delta G$, since apparently the quarks didn't make up for the proton spin and spin physics 'enjoyed' its crisis.

A general impression I got from this quick glance ${ }^{c}$ on historic aspects of HEP and of DIS in particular has been that the development of leptonnucleon physics seems to have followed a rational logic, from Hofstatter's elastic experiments via lepton-nucleon fixed target experiments to HERA. One wishes to trust that this continues as is strongly suggested by the recent results and developments of the field of DIS, many of which were presented at this workshop as summarised in the working group reports, these proceedings, of which only a few can be sketched in the following.

\footnotetext{
cA ten year's 'net' misses important steps which happen in between. Further important results in DIS include the discovery of weak neutral currents in neutrino-nucleon scattering in 1973, the confirmation of the weak isospin of the right handed electron to be zero in polarised ep scattering at low $Q^{2}$ in 1978 and the discovery of an unbalanced proton spin by the EMC experiment in 1988. One furthermore knows that writing history is more than difficult when it regards too recent times.
} 


\section{Results}

\subsection{Spin}

Often spin physics is disregarded in the context of DIS. Yet a simple evaluation reveals its close connection with unpolarised lepton-nucleon scattering. Spin physics measures the $g_{1}$ structure function, $2 g_{1}=\Sigma e_{q}^{2}\left(q^{+}-q^{-}\right)$, where $e_{q}$ is the electric charge of quark $q$ and $q^{+(-)}$denotes the momentum distribution of a quark inside the nucleon with its spin parallel (antiparallel) to the nucleon's spin. The classic structure function $F_{1}$ is nothing but the complementary projection, $2 F_{1}=\Sigma e_{q}^{2}\left(q^{+}+q^{-}\right)$, with $2 x F_{1}=F_{2}$ in the naive Quark Parton Model. Integrated over $x$ the functions $F_{1}, g_{1}$ and $h_{1}$ are related to the vector, the axial-vector and the tensor charge of the nucleon. Here $h_{1}$ denotes the transversity distribution related to the transverse spin of quarks. It is thus not surprising that spin physics has developed similarly to unpolarised DIS, as a testing and remarkable development ground for QCD in the understanding of nucleon structure. Despite its growing data base, including first RHIC results and fragmentation function determinations in $e^{+} e^{-}$, and despite the impressive theoretical progress, the original question as to how partons may compose the proton spin has not been answered yet. The proton spin of $1 / 2$ is composed of the contribution $\Sigma$ from quarks, $\Delta G$ from gluons and orbital momenta $L_{q, g}$ from quarks and gluons ${ }^{d}$. The fact that the quark contribution is too small to add up to $1 / 2$, originally discovered in a $g_{1}$ measurement by the EMC ${ }^{5}$, has been termed a spin crisis as it points to a violation of the OZI rule. This may be resolved if the strange quark contribution $\Delta s$ to the quark spin or $\Delta G$ were large. Recent results from HERMES using a beautiful RICH, though from leading order semi-inclusive data analyses, point to zero strange spin while previous hadron pair data suggest $\Delta G$ to be as large as 0.4 at $x \simeq 0.2$. New data on $\Delta G$ presented at this conference by RHIC and COMPASS, point to a small gluon contribution albeit measured with a large uncertainty which can not exclude $\Delta G$ to finally balance the nucleon's momentum. Resolving this fundamental issue requires a high luminosity polarised $e N$ collider of

\footnotetext{
${ }^{\mathrm{d}}$ Generalised parton distributions are sensitive to the transverse proton spin structure. For example the function $E$ is related to the target spin asymmetry $A_{U T}$, and thus semi-inclusive measurements, in which the fragmentation function may be chiral odd, may allow the angular momentum of quarks to be accessed. Much theoretical effort in phenomenology, perturbative and lattice QCD is devoted to this and first model dependent constraints can be obtained from experimental data as shown by HERMES at this workshop.
} 
variable energy. It has to be a high energy collider to cover a large range of Bjorken $x$ to reliably determine the moment of the polarised gluon distribution in the DIS region. Today's low $x$ spin results are at too small $Q^{2}$ for pQCD to be applicable. The collider has to have high luminosity, $>10^{32} \mathrm{~cm}^{-2} \mathrm{~s}^{-1}$, since the spin asymmetries to be measured are proportional to $x$. Its energy has to be variable, in polarised mode, since one needs to measure $g_{1}$ at different $Q^{2}$ at fixed $x$ while the measurement is sensitive only along a band in the $x, Q^{2}$ plane for which the inelasticity $y$ is large, $y \geq 0.5$. Desirably it should have a deuteron option ${ }^{\mathrm{e}}$ in order to disentangle the up and down flavour contributions to the nucleon's spin. Impressive instrumental progress was reported on the polarised $p p$ machine RHIC, polarimetry and experiments at BNL and new results are expected on the gluon and transverse spin. It has been proposed, also at DIS06, to add a polarised electron ring or linac to RHIC and also to build a high luminosity collider at Jlab. Similar to HERA, which opened a new world in unpolarised DIS, one polarised $e N$ collider would considerably extend the range of the inclusive measurements and allow for a new level of final state measurements in polarised DIS.

\subsection{Electroweak Physics at HERA}

The most important news at DIS06 had been that the luminosity production at HERA II, within the years 2004/05, has now overtaken by a factor of two the integrated luminosity collected at HERA I, from 1992 to 2000, and former serious background problems are overcome. Moreover, HERA now runs in polarised mode, the $e^{ \pm}$beam polarisation reaching values of $\pm 40 \%$. Thus electroweak HERA physics has started to become of real interest. First impressive results have been obtained in essentially four areas: i) the very recent yet classic measurements of the polarisation dependence of the charged current cross section, a unique result which excludes the existence of right handed (r.h.) charged currents up to masses $M_{W_{R}}$ slightly higher than $200 \mathrm{GeV}$; ii) the determinations of the light quark weak neutral current vector and axial vector couplings from NLO QCD fits to the $\mathrm{NC}$ and $\mathrm{CC}$ data by $\mathrm{H} 1$ and ZEUS, leading to results of competetive accuracy to the CDF data, and resolving ambiguities of LEP data in the $a-v$ plane of $u$ and $d$ quarks; iii) the charge asymmetry data from which the

${ }^{\mathrm{e}}$ As noted by Derbenev and Skrinsky, since the anomalous magnetic moment of the deuteron is much smaller than that of the proton, less resonances are to be passed for deuterons and thus a polarised $e d$ collider may be easier to realise than an $e p$ machine. 
interference structure function $x F_{3}^{\gamma Z}=2 x\left[a_{u} e_{u}(U-\bar{U})+a_{d} e_{d}(D-\bar{D})\right]$ can be determined, which is sensitive to the sign of quark charges and to a possible difference between sea quark and antiquark distributions ${ }^{\mathrm{f}}$ for which $\mathrm{NuTeV}$ have indications in their determination of the strange sea asymmetry; iv) the first measurement of a polarisation asymmetry in $\mathrm{NC}$ $e^{+} p$ and in $e^{-} p$ scattering which, combining the data of H1 and ZEUS ${ }^{6}$, demonstrates parity to be violated down to distances as small as $10^{-18} \mathrm{~m}$.

\subsection{Diffractive Progress}

At this workshop new sets of data were presented on inclusive diffraction. Theoretically collinear factorisation has been proven at fixed $x_{I P}$ and $t$, which allows diffractive parton densities to be introduced with the constraint that the proton does not fragment during the violent $e p$ collision. Here $t$ is the 4 -momentum transfer at the proton vertex and $x_{I P}$ denotes the momentum fraction of the proton carried by the diffractive exchange. Data are available which identify the diffractive exchange as the absence of forward activity, "large rapidity gap" (LRG) data, or by tagging the proton downstream, near the proton beam line at $100-200 \mathrm{~m}$ distance, "[V]FPS" (H1) or "LPS" (ZEUS) data. The ratio of the LRG, for $M_{Y}<1.6 \mathrm{GeV}$, to the FPS cross sections is 1.23 which is measured to be constant within the kinematic range of the measurement. Thus the dominant process in the gap data is indeed diffraction and further processes such as resonance or neutron production do not alter the kinematic dependencies beyond the point to point uncertainty of typically $10 \%$ of the LRG/FPS ratio. The detailed H1 NLO QCD analysis assumes an additional factorisation in writing the diffractive parton densities as a product of a flux factor for the diffractive exchange times the parton distributions, $f_{i}\left(\beta, Q^{2}\right), i=q, g$, which describe the inclusive cross section data successfully. Here $\beta=x / x_{I P}$ is the momentum fraction of the diffractive exchange carried by its partons. The accuracy of the now final $\mathrm{H} 1$ data allows the $\ln Q^{2}$ derivative of the reduced cross section to be measured, which is shown in Fig. 1 together with its decomposition in the QCD fit into the quark and gluon part. The result

\footnotetext{
${ }^{\mathrm{f}}$ Measurement of this function requires highest $Q^{2}$ and large $y$. It can be measured much better at the LHeC, see below, than at HERA due to the much increased kinematic range and the superior luminosity. Thus, at the LHeC, one will be able to access the behaviour of valence quarks down to $x$ as small as $10^{-3}$ and possibly with deuteron data measure the difference of sea and anti-quarks for up $(U)$ and down $(D)$ quarks down to extremely small $x$.
} 


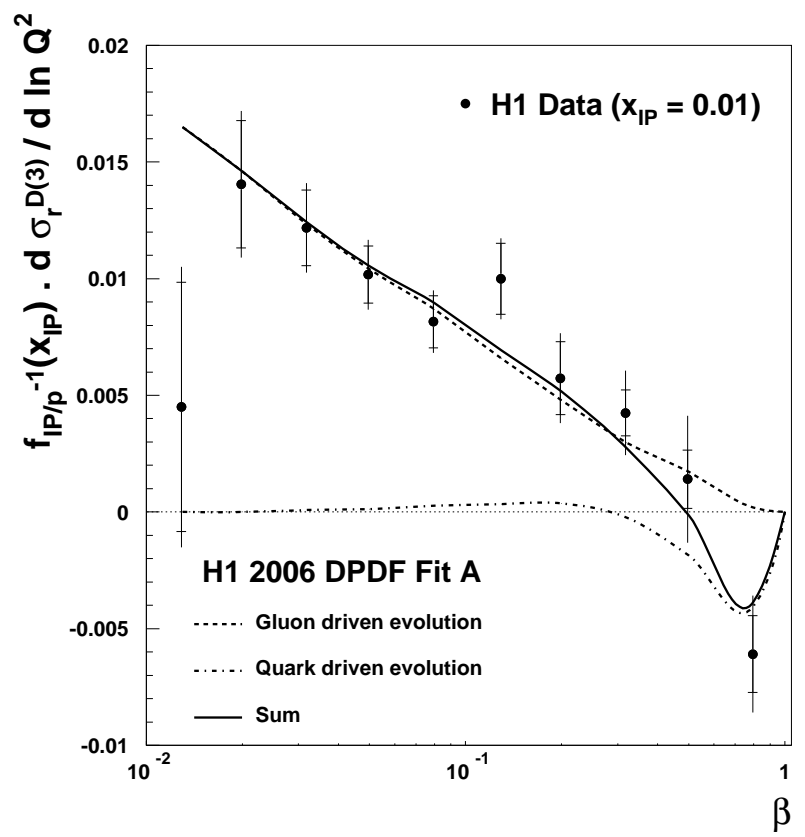

Figure 1. Derivative of the reduced diffractive cross section at $x_{I P}=0.01$ and its decomposition into the gluon and quark parts in the H1 NLO QCD analysis.

reveals that the cross section evolution for small $\beta<0.4$ is gluon driven, much as the inclusive proton dynamics in the DIS region is dominated by pair production from gluons ${ }^{\mathrm{g}}$. The logarithmic derivatives of $\sigma_{r}^{D}$ and $\sigma_{r}$ with respect to $\ln Q^{2}$ are measured to be the same for $\beta<0.6$. Thus low $\beta$ diffraction and low $x$ inclusive scattering seem to reflect a common origin, the dynamics of the QCD vacuum. It so appears less surprising that a number of detailed, recent observations in diffractive DIS resemble inclusive DIS such as the rise towards low $\beta$ or $x$ of the $\ln Q^{2}$ derivatives or the common fraction of charm of about $20 \%$, away from thresholds, in both the diffractive cross section, as presented to this workshop, and in the inclusive cross section. Fig. 1 also reveals why a sole fit to inclusive diffractive data, again similarly to inclusive scattering, fails to constrain the gluon density well at large $\beta$ : this region is sensitive to quarks, i.e. gluon bremsstrahlung,

${ }^{g}$ Wilzcek calls the observation at HERA that the proton viewed at small dimensions is glue the verification of one of "the most dramatic consequences" from pQCD. 
only. H1 thus chose to present two inclusive fits of very similar quality which have a very similar quark singlet distribution, as both have to describe the $F_{2}^{D, 3}$ data, but a larger (A) or lower (B) gluon distribution at $\beta>0.4$. Confronting this behaviour with new diffractive di-jet data, as presented by $\mathrm{H} 1$ to this workshop, prefers the gluon distribution at large $\beta$ to be rather low. Diffraction has thus evolved from the first surprise of its presence at HERA to a rather exact and interesting testing ground of perturbative QCD to which further data from both HERA collaborations and studies of universality in comparing $e p$ predictions with Tevatron and later LHC data will certainly contribute a great deal. Diffraction is recently considered as a possible means of discovering the Higgs particle in a clean, double diffractive scattering environment $p p \rightarrow p H p$. Events of similar type, as reported to this workshop, were recently observed at the Tevatron. This line is followed in the FP420 project at the LHC with the hope that cross sections as in SUSY theories may be large enough to give a chance of observing diffractive Higgs production at foreseeable luminosities, time scales and using ingenuitive triggers.

\subsection{Beauty}

The physics of $b$ quarks is investigated only at the $B$ factories, one might think. This does not hold for the production of $b$ quarks, the mechanism of which is investigated in detail at the Tevatron and at HERA. Interesting results on beauty, and charm, fragmentation functions are obtained in theory and in $e^{+} e^{-}, p \bar{p}$ and $e p$ experiments. A large part of the data is obtained around threshold and evolved theoretical work is ongoing to correctly describe the production and the fragmentation in this region which is important to correctly predict heavy quark density evolution or to extract a gluon distribution. The beautyful highlight of recent $B$ physics, not only at this workshop, has been the measurement by D0 and CDF of the oscillation frequency $\Delta m_{s}$ of $B_{s}-\bar{B}_{s}$ mixing based on an integrated luminosity of $1 \mathrm{fb}^{-1}$ in each experiment. Beyond the genuine importance of this measurement for understanding the mechanism of $\mathrm{CP}$ violation, these results demonstrate the capability of a hadron collider to function in $B$ factory "mode", the $\Delta m_{s}$ result of CDF being accurate to about $3 \%$ statistically and $0.4 \%$ systematically.

HERA has begun to measure the beauty structure function $F_{2}^{b \bar{b}}$, which defines the beauty "contents" of the proton or its radiative production near and below threshold, see Fig. 2. The accurate knowledge of the $b$ quark 


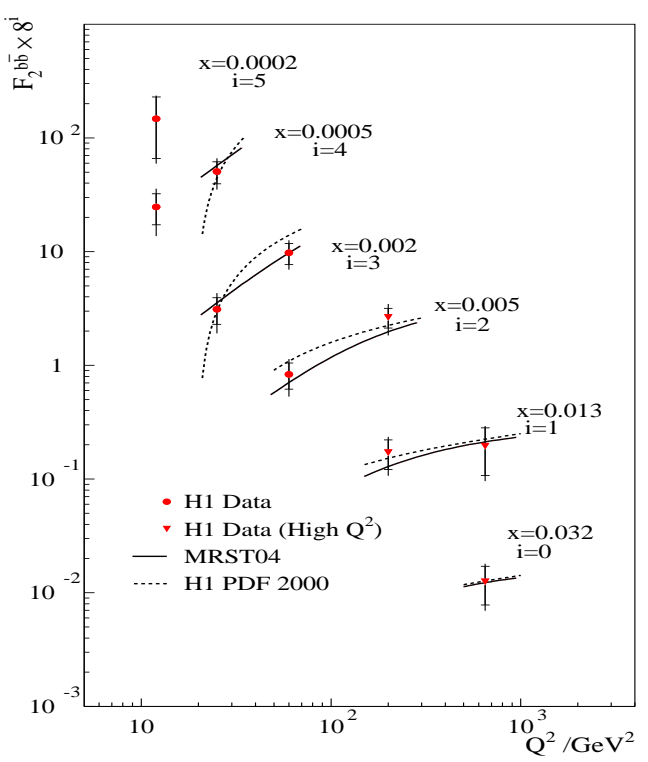

Figure 2. Measurement of the beauty structure function $F_{2}^{b \bar{b}}$ as a function of $Q^{2}$ at low $x$, from a Silicon vertex detector based impact parameter analysis of H1's HERA I data, compared with NLO QCD fits of variable flavour, by MRST, and of fixed flavour, by H1. It is clearly seen that beauty is produced below the threshold $Q^{2}=m_{b}^{2}$. With more data being taken by both $\mathrm{H} 1$ and ZEUS one can envisage this measurement to become as accurate as about $10 \%$, for a quantity which amounts to only a few $\%$ of the inclusive DIS cross section at HERA.

density is important for testing QCD and as a tool for discoveries at the LHC. The beauty contribution to the total $Z$ production cross section at the LHC amounts to about $5 \%$. It thus needs to be measured at HERA with an accuracy of $10-20 \%$ in order not to dominate the $Z$ cross section prediction uncertainty which one hopes to determine at the per cent level. The $b$ quarks will play an extensive role at the LHC, in the investigations of parton dynamics and in the searches for new physics, for example in the gluon-gluon Higgs production, $g g \rightarrow b H \bar{b}$ or $g b \rightarrow H b$. Its role will also depend on the mechanisms realised at $\mathrm{TeV}$ scales. For example, in the SM, Higgs production is dominated by $g g \rightarrow H$ and $b \bar{b} \rightarrow H$ is negligible. At large $\tan \beta$, however, in the MSSM beauty is larger than gluon Higgs production ${ }^{8}$. If one scatters protons off protons one has to accurately know the partonic nature of the projectiles which requires precise $e p$ data. 


\subsection{Parton Radiation at Low $x$}

HERA provides phase space in $x$ and transverse parton momentum $k_{t}$ which allows the mechanism of gluon radiation at low $x$ to be studied in detail. In the low $x$ DIS region, the gluon density is high. A wealth of data has been investigated in order to find deviations from the $k_{t}$ ordered DGLAP prescription and contribute to the development of low $x$ theory. Recent analyses of $\mathrm{H} 1$ and ZEUS suggest that DGLAP theory in NLO may fail in the description of the emission of jets in the forward (outgoing proton beam direction) at low $x$ and $Q^{2}$. Hints for a breakdown of the conventional theory come also from the study of multi-jets, as presented to this workshop, and azimuthal correlations between dijets, which at low $x$ and $Q^{2}$ seem to be weaker than predicted in NLO DGLAP theory. Firm interpretations of these observations are subject to the uncertainties connected with yet higher order pQCD contributions, as mostly departures from DGLAP QCD are seen in regions of large NLO effects compared to LO. They are also related to effects of resolved photon structure. "Unintegrated", $k_{t}$ dependent parton distributions are being introduced which may allow a more accurate description of the final state as they incorporate transverse momentum kinematic effects in their definition. Unintegrated parton distributions are of increasing importance also in the description of diffractive vector meson production. Theoretically resummations may rescue DGLAP theory at HERA. Yet higher accuracy, of inclusive and final state data, and a substantial extension of the kinematic range, as would be possible with the LHeC, are essential for further progress of this field. Parton densities are expected to be amplified $\propto A^{1 / 3}$ in a nuclear environment which relates an $A A$ programme, as with ALICE, to the $e A$ option of the LHeC. Meanwhile Lev Lipatov develops the BFKL Pomeron in string theory.

\subsection{Possible Discoveries at the TeVatron or at HERA}

Could the TeVatron still find the Higgs prior to the LHC? As presented this year and to this workshop, the answer is "may be". This could happen if the Higgs is there, the TeVatron reaches its anticipated luminosity, which now ranges between what is called a base and a design goal, from $4-8 \mathrm{fb}^{-1}$ by the end of 2008, the physicists go for it, cross the D0 and CDF and decay channel borders and don't leave all to the LHC, which has a much further reaching goal but requires a new machine and huge detectors and collaborations to function well in this decade and much beyond.

HERA, as the TeVatron, is a machine operating at the energy frontier. 
Thus a strong effort is made on both machines to search for physics beyond the Standard Model. Limits have been set at several hundreds of $\mathrm{GeV}$ up to $\mathrm{TeV}$ scales in indirect analyses, for example in searches for contact interactions, leptoquarks, extra dimensions or supersymmetric particles. An intriguing peculiarity at HERA is the events in which the final state contains an isolated lepton, large missing transverse momentum and a hadronic system with a large transverse energy, which are regularly observed by the H1 Collaboration in $e^{+} p$ scattering, at an excess rate of 3.4 standard deviations from $158 \mathrm{pb}^{-1}$ of integrated luminosity corresponding to $1 e$ or $\mu$ event per $10 \mathrm{pb}^{-1}$. The data still to be taken are expected to shed further light on this observation, which currently is the largest deviation from the standard model observed at large scales at HERA.

\section{Theory}

In 1955 F. Dyson wrote "to summarise a theoretical session is almost a contradiction in terms" and selected two to three topics. At this workshop major theoretical activities and results were presented and are summarised in the working group reports. Perhaps a few remarks to theory are in order beyond a general acknowledgment of the huge theoretical efforts, in perturbative QCD, lattice QCD, phenomenology, field theory etc., without which we could not interpret or reliably analyse the data. There is firstly the increasing accuracy of the data at HERA, owing to the high precision detectors and redundant energy calibration techniques as well as increased statistics, which contrasts with what is called the "scale uncertainty" of QCD due to the renormalisation group. An example is the strong coupling constant which is the least well-measured of the fundamental coupling constants and thus dominates the uncertainty of extrapolations of the electromagnetic, weak and strong coupling constants to a unification scale near the Planck mass. The combined $\alpha_{s}\left(M_{Z}^{2}\right)$ value to NLO from ZEUS and $\mathrm{H} 1$ as determined in inclusive DIS and in jet production currently is ${ }^{7}$ $\alpha_{s}=0.1186 \pm 0.0011(\exp ) \pm 0.005($ thy $)$. Here the first uncertainty comprising all experimental and model dependent effects is already smaller than the current world average error. A striking peculiarity of this result is the so-called theoretical error. Its size reflects the ad hoc convention that the renormalisation (and factorisation) scale should be varied by factors of 2 and $1 / 2$. This convention is not supported by the data: in both the $\mathrm{H} 1$ and the ZEUS inclusive NLO QCD analyses, fits are very poor at the extremes of these scale variations and thus the variation prescription is questionable. 
With forthcoming exact NNLO analyses the scale dependence will be further reduced but the arbitrariness of the scale choice remains problematic. A similar example is dijet cross section data, which e.g. for ZEUS have a theoretical "error" of up to $20 \%$ which is the largest of all. The variation procedure of $0.5 \ldots 2 \mu_{R}$ was introduced in the $80^{\text {th }}$ as a means of comparing the sensitivity of various processes to higher orders. Such a sensitivity is inherent to the renormalisation group. Yet, its size prescription is totally ad hoc and if progress is to be made it needs to be revisited in the light of knowing the NNLO corrections and comparing processes and schemes. A coordinated theoretical re-assessment is very much desired from an experimenter's point of view as the data produced by hundreds of physicists get devalued by the present procedure. It has been proposed to ignore the scale dependence, not a too popular point of view and scheme, and to use intelligence in stating what such a theoretical error means, not too popular a suggestion either. A new accord is required. From a phenomenological point of view, given the observations in HERA data analyses, it seems justified to replace $1 / 2 \ldots 2 \mu_{R}$ by $1 / \sqrt{2} \ldots \sqrt{2} \mu_{R}$ : this has already been used, the fits are stable in this reduced range and the resulting variation, which is $\simeq 0.002$ for $\alpha_{s}\left(M_{Z}^{2}\right)$, comes out to be comparable to the difference of $\alpha_{s}\left(M_{Z}^{2}\right)$ determined in NLO and NNLO. The problem is not straightforward ${ }^{9}$.

A further remark regards multi-jet production to which enormous efforts are directed as reported at this workshop. Multi-jet production carries important information on parton dynamics. The dynamics studied in multi jet production at low $x$ at HERA ${ }^{10}$ are essential for a large, the forward part of LHC physics. At the LHC one may expect about 200 events per $\mathrm{fb}^{-1}$ in which eight jets are produced. In this regard one hopes for further progress in simplifying (?) the theory by the introduction of recursion relations between gluon amplitudes ${ }^{11}$, in which a multi emission amplitude is related to the sum of propagator relations between sub amplitudes, of lesser multiplicity. Similarly impressive have been the theory and implementation of new or further developed simulation programs, such as MC@NLO, ThePEG, Ariadne, PYTHIA, CASCADE, which are the basis for understanding current and hopefully future measurements. We have not seen instantons, odderons or axions, QCD theory can be ahead of experiment. 


\section{Outlook}

While the physics of DIS expands rapidly its main current basis HERA will be terminated in 2007. This is to be regretted but may also be seen as the strongest possible hint for the community to unite behind the possible next big steps. These lead into a new area of spin physics as discussed but fundamentally require a $\mathrm{TeV} e p$ collider of the highest possible luminosity and energy. The $\mathrm{LHeC}^{3}$ promises to have it all, the kinematic range, the high luminosity, the modest cost, on the LHC and ILC scales, the complementarity to the LHC, the guaranteed high precision parton dynamics, the high density physics beyond unitarity and the potential of investigating how electrons and quarks possibly form resonances. It is thus worth a strong effort to have it moved forward to when, around 2009, the international HEP community needs to define its next steps. The field of deep inelastic scattering as summarised in Fig. 3 did fundamentally contribute to the understanding of the structure of nature and to the development of the standard model in the past. It must be brought sooner rather than later to the $\mathrm{TeV}$ level of energy which is required to have $e p$ data when the SM model likely gets surpassed by new physics. The future of collider particle physics requires not "just" the LHC, not "only" the ILC, it needs a balanced programme with $p p, e^{+} e^{-}$and $e p$ machines much like it was realised at the $10 \mathrm{GeV}$ level and at the Fermi scale by the former fixed target leptonnucleon experiments and HERA, respectively, and their hadron-hadron and electron-positron counterparts.

Acknowledgments I would like to thank the organisers for the invitation to this talk and Paul Newman for carefully reading the manuscript. May Mark Strikhman forgive that I didn't discuss the first measurement of the transverse size of the gluon. Apologies for not having the space for proper citations and for a rather personal selection of subjects from the so many contributions to the workshop. Thanks to our Japanese colleagues, DIS06 at Tsukuba was a remarkable success and great experience.

\section{References}

1. C.H. Llewellyn Smith, "Physics at Future High Energy Colliders", Proc. XXIII ICHEP Conference, Berkeley, California (1986).

2. A. Caldwell, these Proceedings.

3. J.Dainton et al., "Deep Inelastic Scattering at the LHC", DESY-06-006, Cockcroft-06-05, JINST to appear; see also J. Dainton these Proceedings.

4. T. Alexopoulos et al., "eD Scattering with H1", LoI, DESY 03-194;

H. Abramowicz et al., "A New Experiment for HERA", MPP-2003-62;

F. Willeke and G. Hoffstaetter, Talks at the Workshop on the Future of DIS, 


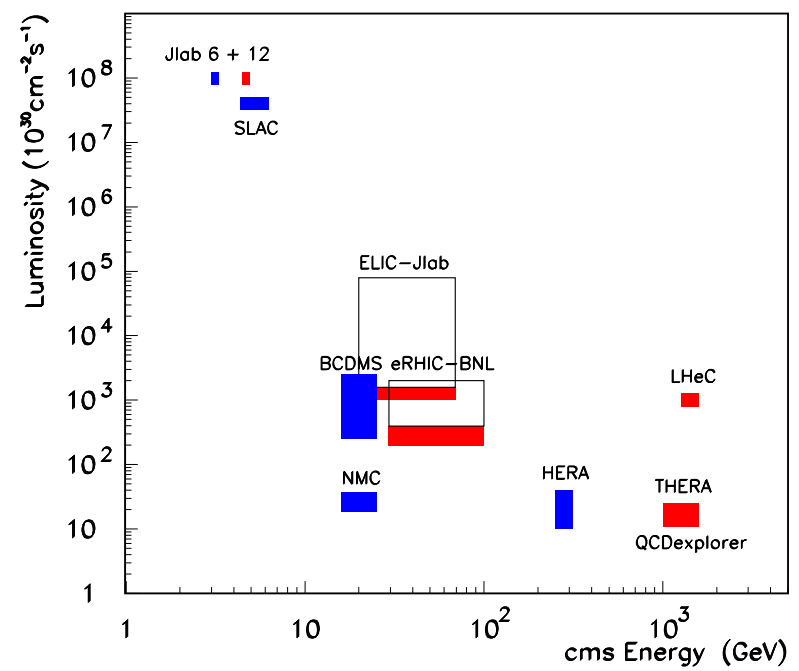

Figure 3. Energy and luminosity for existing (dark, blue boxes) and prospects for new (grey, red boxes) facilities in unpolarised lepton-proton DIS. The SLAC box indicates the luminosity of the pioneering ep experiment. The Jlab fixed target $12 \mathrm{GeV}$ upgrade programme, as also presented at this workshop, is directed to very high statistics physics at low $Q^{2}$ and large $x$. BCDMS and NMC have provided the most accurate DIS muonproton structure function data using $30 \mathrm{~m}$ and $3 \mathrm{~m}$ long unpolarised hydrogen targets, respectively. The large luminosities envisaged at eRHIC and ELIC (hollow boxes) for polarised $e p$ physics are based on energy-recovery linac technology. HERA has reached peak luminosities of up to $5 \cdot 10^{31} \mathrm{~cm}^{-2} \mathrm{~s}^{-1}$ with its luminosity upgrade. The LHeC is designed for the highest energy and largest luminosity. Linac-ring accelerator designs as for THERA ${ }^{12}$ (TESLA/ILC-HERA) and the QCD explorer ${ }^{13}$ (CLIC-LHC) have lower luminosity estimates.

Durham 2001, unpublished.

5. J. Ashman et al., EMC Collaboration, Phys. Lett. B 206 (1988) 364.

6. H1 and ZEUS Collaborations, "Electroweak Neutral Currents at HERA", H1-prel-06-142, ZEUS-prel-06-022, contributed to ICHEP06, presented by V. Chekelyan, the first ever joint $\mathrm{H} 1$ and ZEUS paper.

7. C. Glasman, hep-ex/0506035.

8. A. Belyaev, J. Pumplin, W.K. Tung and C.P. Yuan, JHEP 0601 (2006) 069.

9. S. Brodsky, K. Ellis, W.van Neerven and D. Soper, private communications.

10. F. Eisele, Talk at ICHEP06, Moscow, 2006, to be published.

11. R. Britto, F. Cachazo and B. Feng, Nucl. Phys. B 715 (2005) 499.

12. The THERA Book, "ep Scattering at $\sqrt{s} \sim 1 \mathrm{TeV}$ ", DESY 01-123F, eds. U. Katz, M. Klein, A. Levy and S. Schlenstedt.

13. D. Schulte and F. Zimmermann, "QCD Explorer based on LHC and CLIC-1", CERN AB-2004-079, CLIC Note 608; L. Gladilin et al., hep-ph/0504008. 